\title{
Emergence of Azole-Resistant Aspergillus fumigatus Strains due to Agricultural Azole Use Creates an Increasing Threat to Human Health
}

\author{
Anuradha Chowdhary ${ }^{1 *}$, Shallu Kathuria ${ }^{1}$, Jianping $\mathrm{Xu}^{2}$, Jacques F. Meis ${ }^{3,4}$ \\ 1 Department of Medical Mycology, Vallabhbhai Patel Chest Institute, University of Delhi, Delhi, India, 2 Department of Biology, McMaster University, Hamilton, Ontario, \\ Canada, 3 Department of Medical Microbiology and Infectious Diseases, Canisius Wilhelmina Hospital, Nijmegen, The Netherlands, 4 Department of Medical Microbiology, \\ Radboud University Nijmegen Medical Centre, Nijmegen, The Netherlands
}

Aspergillus fumigatus, a ubiquitously distributed opportunistic pathogen, is the global leading cause of aspergillosis and causes one of the highest numbers of deaths among patients with fungal infections [1]. Invasive aspergillosis is the most severe manifestation with an overall annual incidence up to $10 \%$ in immunosuppressed patients, whereas chronic pulmonary aspergillosis affects about 3 million, primarily immunocompetent, individuals each year [2]. Three triazole antifungals, namely itraconazole, voriconazole, and posaconazole, are recommended first-line drugs in the treatment and prophylaxis of aspergillosis [3]. However, azole resistance in $A$. fumigatus isolates is increasingly reported with variable prevalence in Europe, the United States, South America, China, Japan, Iran, and India [4-9]. For example, about 10\% of strains of $A$. fumigatus from the Netherlands are itraconazole resistant, and in the United Kingdom, the frequency increased from $0 \%-5 \%$ during $2002-2004$ to $17 \%-20 \%$ in 2007-2009 [1013]. In the ARTEMIS global surveillance program involving 62 medical centers, $5.8 \%$ of $A$. fumigatus strains showed elevated MICs to one or more triazoles [5]. Similarly, the prospective SCARE (Surveillance Collaboration on Aspergillus Resistance in Europe) study involving 22 medical centers in 19 countries identified an overall prevalence of $3.4 \%$ azole resistance. Azole-resistant $A$. fumigatus (ARAF) ranged from $0 \%$ to $26 \%$ among the 22 centres and was detected in 11 (57.9\%) of the 19 participating European countries [4 and P.E. Verweij, personal communication]. Interestingly, almost half $(48.9 \%)$ of the ARAF isolates from the SCARE network in European countries were resistant to multiple azoles and harbored the $\mathrm{TR}_{34} / \mathrm{L} 98 \mathrm{H}$ mutation in the cyp51A gene [4 and P.E. Verweij, personal communication]. Indeed, multiazole resistance in $A$. fumigatus due to the $\mathrm{TR}_{34} / \mathrm{L} 98 \mathrm{H}$ mutations has become an emerging problem in both Europe and Asia and has been associated with high rates of treatment failures [12-14].

Azole antifungal drugs inhibit the ergosterol biosynthesis pathway, specifically the cytochrome p450 sterol 14 - $\alpha$-demethylase encoded by the cyp51A gene, which leads to depletion of ergosterol and accumulation of toxic sterols. The majority of ARAF isolates contain alterations in the target enzyme and the mutated target showed reduced or no binding to the drugs [15]. While most mutations in ARAF isolates were single nucleotide substitutions in the target gene (cyp51A), mutations at other genes such as the $c d r 1 \mathrm{~B}$ have also been reported. For example, in the United Kingdom the frequency of ARAF isolates without cyp51A mutations has been reported to be more than $50 \%$ [16].

\section{Routes of Azole Resistance Development}

The epidemiologic data on azole resistance is mainly from two clinical entities. One group comprises noninvasive diseases including patients with allergic bronchopulmonary aspergillosis (ABPA), aspergilloma, and chronic pulmonary aspergillosis (CPA) who were treated with long-term azole therapy (mainly itraconazole) and developed acquired resistance after 1-30 months of treatment [13]. In these patients, the ARAF isolates may be resistant to only itraconazole or exhibit a multi-azole-resistant phenotype. The underlying resistance mechanism commonly involves point mutations in the cyp51A gene, indicating that in patients exposed to long-term azole therapy, the fungus is capable of rapidly adapting to azole drug(s) [11-14]. The genotypic analysis of serial isolates of $A$. fumigatus from patients with chronic aspergillosis revealed that the initial susceptible and later resistant isolates had the same genotype. The only changes were the specific mutations conferring azole resistance, consistent with the development of resistance arising from azole therapy [13].

The second group of patients with ARAF are those with acute aspergillosis but with no known prior exposure to azole drugs [12]. In contrast to the first group in which de novo mutation of the fungus in cavitary lesions is the primary mechanism for the development of azole resistance, those of the second group likely acquired ARAF strains from external environments. In fact $50 \%$ of the patients with invasive aspergillosis due to ARAF are known to be azole naive and the outcome of patients with azole-resistant invasive aspergillosis has been dismal, with a mortality rate of $88 \%$ [12]. Eighty percent of the ARAF strains from patients with invasive aspergillosis described in the SCARE network had the $\mathrm{TR}_{34} / \mathrm{L98H}$ mutations, which consist of a substitution of leucine to histidine at codon 98 of the cyp51A gene in combination with a 34-bp tandem repeat in the promoter region. These mutations enabled resistance to itraconazole and intermediate susceptibility or resistance to voriconazole, posaconazole, or both $[4,17,18]$. As described above, although the environmentally derived azoleresistant strains are predominately associated with acute invasive infections $[12,19]$, the same mechanism has also been reported in

Citation: Chowdhary A, Kathuria S, Xu J, Meis JF (2013) Emergence of AzoleResistant Aspergillus fumigatus Strains due to Agricultural Azole Use Creates an Increasing Threat to Human Health. PLoS Pathog 9(10): e1003633. doi:10.1371/ journal.ppat.1003633

Editor: Joseph Heitman, Duke University Medical Center, United States of America

Published October 24, 2013

Copyright: (C) 2013 Chowdhary et al. This is an open-access article distributed under the terms of the Creative Commons Attribution License, which permits unrestricted use, distribution, and reproduction in any medium, provided the original author and source are credited.

Funding: The authors received no specific funding for this study.

Competing Interests: JFM received grants from Astellas, Basilea, and Merck. He has been a consultant to Astellas, Basilea, and Merck and received speaker's fees from Merck and Gilead. All other authors have declared that no competing interests exist. This does not alter our adherence to all PLOS Pathogens policies on sharing data and materials.

* E-mail: dranuradha@hotmail.com 
patients with chronic and allergic pulmonary infections [20]. For example, Denning et al. detected $\mathrm{TR}_{34} / \mathrm{L} 98 \mathrm{H}$ and $\mathrm{M} 220$ mutations in $55.1 \%$ respiratory samples of CPA and ABPA patients by direct PCR and some of these patients had no prior azole therapy [20].

\section{Environmentally Mediated Development of Azole Resistance}

Several recent findings support the hypothesis that ARAF strains in patients with invasive aspergillosis were more likely to be acquired from environmental sources rather than from de novo mutation and selection within patients during azole therapy. First, ARAF strains have been found in patients who had never been treated with azole antifungal drugs [7,12-14]. Second, ARAF strains have been found in many environmental niches including flowerbeds, compost, leaves, plant seeds, soil samples of tea gardens, paddy fields, hospital surroundings, and aerial samples of hospitals [19,21-23]. The majority of the environmental ARAF isolates harbor the $\mathrm{TR}_{34} / \mathrm{L} 98 \mathrm{H}$ mutations at the cyp51A gene [19,21-23]. ARAF isolates with the $\mathrm{TR}_{34} / \mathrm{L} 98 \mathrm{H}$ mutations have been detected in the environment of the Netherlands, Denmark, India, and Iran [19,21-23]. It is noteworthy that environmental surveys of ARAF from Europe reported that $12 \%$ of Dutch soil samples and $8 \%$ of Danish soil samples had the $\mathrm{TR}_{34} / \mathrm{L} 98 \mathrm{H}$ genotype $[19,22]$. Similarly, environmental surveys across India detected that $7 \%$ of all $A$. fumigatus isolates and $5 \%$ of soil/aerial samples carried the $\mathrm{TR}_{34} / \mathrm{L} 98 \mathrm{H}$ mutation [21]. These strains showed cross-resistance to voriconazole, posaconazole, itraconazole, and to six triazole fungicides used extensively in agriculture [21].

Recently, another mutation of the cyp51A gene $\left(\mathrm{TR}_{46} / \mathrm{Y} 121 \mathrm{~F} /\right.$ T289A) was reported in ARAF isolates from 15 patients in six hospitals in the Netherlands [24]. Interestingly, isolates with the same $\mathrm{TR}_{46} / \mathrm{Y} 121 \mathrm{~F} / \mathrm{T} 289 \mathrm{~A}$ mutations were also recovered from patients' homes and backyards [24]. Apart from the Netherlands, clinical and environmental strains of $A$. fumigatus carrying the $\mathrm{TR}_{46} / \mathrm{Y} 121 \mathrm{~F} / \mathrm{T} 289 \mathrm{~A}$ mutations have also been identified in neighboring Belgium [25] and in India [26]. As most patients acquire $A$. fumigatus from the environment, the emergence and spread of azole-resistant strains in the environment will put more humans at risk, especially those with compromised immunity.

\section{Linking Clinical Azole Resistance in Aspergillus fumigatus to Fungicide Usage in Agriculture}

The hypothesis that clinical azole resistance in A. fumigatus is related to the use of fungicides in agriculture was first proposed by investigators from the Netherlands [19]. The resistant genotype $\mathrm{TR}_{34} / \mathrm{L} 98 \mathrm{H}$ was found in $90 \%$ of $\mathrm{ARAF}$ isolates obtained from azole-naïve patients [11] and they hypothesized that if strains of $A$. fumigatus received sufficient azole challenge in the environment through nonmedical application of azole compounds, azoleresistant strains would be selected and spread [27]. Demethylase inhibitors (DMIs) including azole fungicides are commonly used for crop protection and for the preservation of a variety of materials such as wood [27]. For example, azole fungicides are broadly used to control mildews and rusts of grains, fruits, vegetables, and ornamentals; powdery mildew in cereals, berry fruits, vines, and tomatoes; and several other plant pathogenic fungi. Over one-third of total fungicide sales are azoles (mostly triazoles) and over $99 \%$ of the DMIs are used in agriculture. In addition, there are over 25 types of azole DMIs for agricultural uses, far more than the three licensed medical triazoles for the treatment of aspergillosis. Furthermore, the azoles could persist and remain active in many ecological niches such as agricultural soil and aquatic environments for several months.

The widespread application of triazole fungicides and their persistence in the environment are significant selective forces for the emergence and spread of ARAF. These environmental triazoles can reduce the population of azole-susceptible strains and selecting for azole-resistant genotypes [27]. Intensive use of DMI fungicides for post-harvest spoilage crop protection against phytopathogenic molds is known to cause the development of resistance in many fungi of agricultural importance. For example, resistance or tolerance to triazole fungicides has been reported for important crop pathogens such as Mycosphaerella graminicola (wheat), Rhynchosporium secalis (barley), and Botrytis cinerea (strawberry) [28]. Since $A$. fumigatus shares its natural environments with many fungal plant pathogens, strains of $A$. fumigatus are also exposed to the same strong and persistent pressure from fungicides. Indeed, the presence of a tandem repeat at the $5^{\prime}$-end upstream of the 14- $\alpha$ demethylase gene is an important mechanism found in many plant pathogenic molds resistant to sterol DMI fungicides [19].

All $A$. fumigatus isolates with the $\mathrm{TR}_{34} / \mathrm{L} 98 \mathrm{H}$ mutations from both clinical and environmental origins have shown crossresistance to not only all three medical triazoles, but also five agricultural triazole DMI fungicides: propiconazole, bromuconazole, tebuconazole, epoxiconazole, and difenoconazole [21,29]. These results are consistent with the hypothesis that exposure of $A$. fumigatus to azole fungicides in the environment causes crossresistance to medical triazoles. By molecular modeling studies, these five triazole DMI fungicides were found to have similar molecular structures as medical triazoles (Figure 1) and they all adopt a similar conformation while docking the target enzyme in susceptible strains of $A$. fumigatus $[15,29]$. However, there was limited docking and activity against ARAF strains with the $\mathrm{TR}_{34}$ / L98H mutation [29]. A similar phenomenon was also observed in the maize anthracnose fungus Colletotrichum graminicola. Strains of $C$. graminicola were able to efficiently adapt to media containing azoles, and those adapted to tebuconazole were less sensitive to all tested agricultural and medical azoles than the nonadapted control strain [28]. In addition, tebuconazole induced tandem repeat expansion in the promoter region of cyp51A in A. fumigatus isolates in vitro, indicating that fungicide pressure can rapidly select adaptive genomic changes in this mold [29]. Agriculturally selected antibiotic resistance has also been reported in many bacteria, contributing to the broad distribution of multidrugresistant pathogenic bacteria in patients and hospitals. While horizontal gene transfer is an important mechanism for the spread of antibiotic-resistant genes among bacteria, this mechanism is not commonly found in fungal pathogens. However, similar to that in bacterial pathogens, once multidrug-resistant genotypes arise in fungal pathogens, such genotypes can spread very quickly to other geographic regions and ecological niches through vegetative cells and airborne spores such as conidia.

\section{Clonal Expansion and Fitness of $\mathrm{TR}_{34} / \mathrm{L} 98 \mathrm{H}$ Aspergillus fumigatus Strains}

A recent analysis of 255 Dutch A. fumigatus isolates using 20 molecular markers identified five distinct genotype groups in the Netherlands. Interestingly, all the multi-triazole resistant (MTR) isolates with the $\mathrm{TR}_{34} / \mathrm{L} 98 \mathrm{H}$ mutation belonged to one group and, overall, they were genetically less variable than susceptible isolates [30], consistent with a single and recent origin of the resistant genotype. Similarly, all MTR A. fumigatus clinical and environmental strains obtained from diverse geographical regions from India belonged to a single multilocus microsatellite genotype 

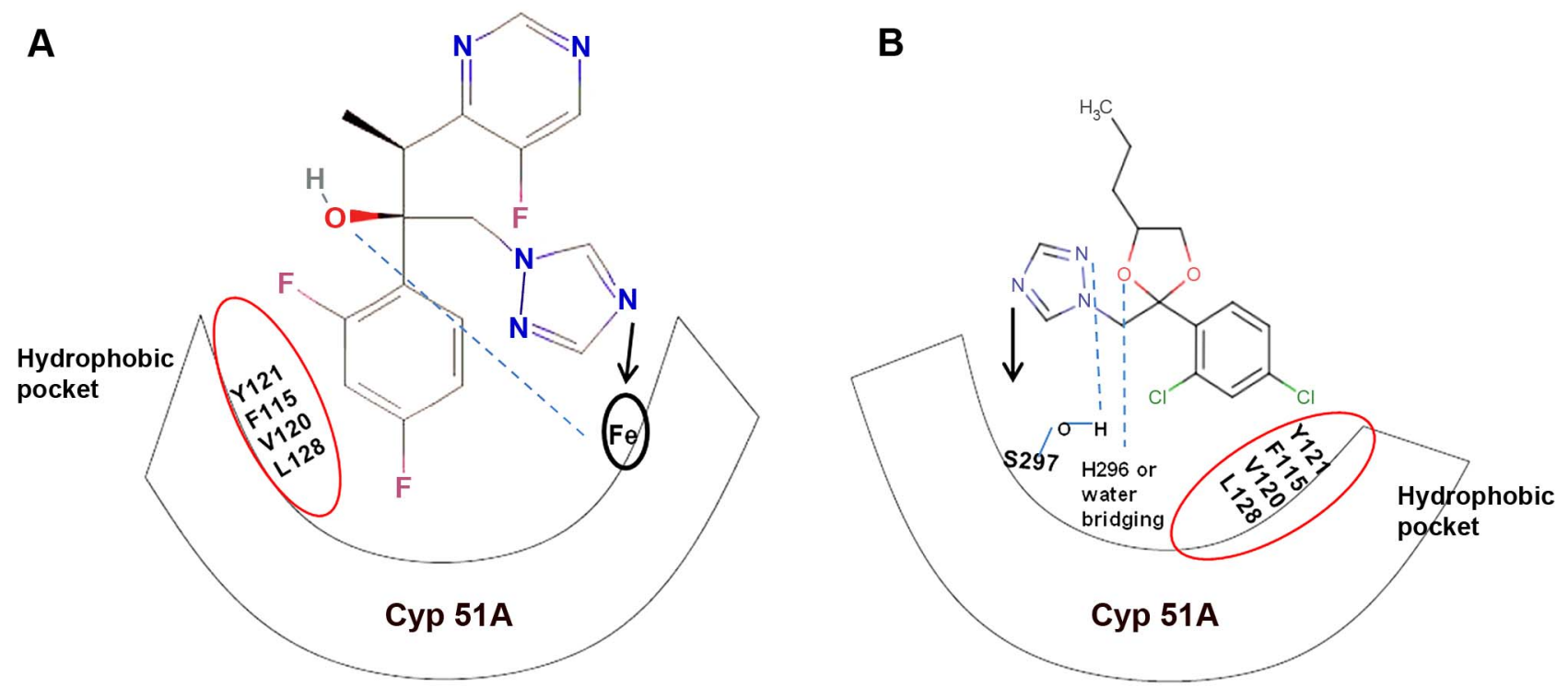

Figure 1. Diagrammatic representation of similar structural binding mode of medical triazoles and triazole fungicides to cyp51A of wild-type $\boldsymbol{A}$. fumigatus. (a) Dihalogenated phenyl group of triazoles forms van der Waals contact with the hydrophobic residues (encircled in red) of the active site $(\operatorname{cyp} 51 \mathrm{~A})$, and the nitrogen atom of the five-membered aromatic ring of triazoles binds to the cyp51A heme moiety. In addition, the Dring propionate $\left(\mathrm{C}_{2} \mathrm{H}_{5} \mathrm{COO}^{-}\right)$of the heme moeity forms hydrogen bonds with the side-chain hydroxyl group of triazoles. (b) Triazole fungicides show similar van der Waals contact at the hydrophobic pocket. However, the nitrogen atom of the five-membered aromatic ring of fungicide triazoles binds to the Ser297 residue at the active site. In addition, the triazoles tebuconazole and epoxiconazole are known to interact with the His296 residue while penconazole and metconazole form water-bridging interactions at the active site. doi:10.1371/journal.ppat.1003633.g001

[21]. The genotype analysis suggested that the ARAF genotype in India was likely an extremely adaptive recombinant progeny derived from a cross between azole-resistant strains migrated from outside of India and a native azole-susceptible strain from within India, followed by mutation. The abundant phylogenetic incompatibility is consistent with sexual mating in natural populations of this species in India [21].

A potential consequence of harboring the multidrug-resistant mutations in ARAF strains might be a reduced fitness in the absence of the drug as compared to the wild-type isolates. However, evidence so far suggested that the $\mathrm{TR}_{34} / \mathrm{L} 98 \mathrm{H}$ mutation had little or no adverse fitness consequence. For example, the composite survival index (CSI) was used to measure the virulence properties of the cyp51A gene-associated resistance mechanism in $A$. fumigatus isolates [31]. The analyses revealed that strains with the $\mathrm{TR}_{34} /$ $\mathrm{L} 98 \mathrm{H}$ mutation had virulence comparable to the wild-type controls and there was no growth impairment and no reduction of virulence with the $\mathrm{TR}_{34} / \mathrm{L} 98 \mathrm{H}$ mutation [31]. A similar finding was reported about the fitness of azole-resistant C. albicans strains with mutations in the ERG3 gene, with the mutants retaining filamentation and virulence properties [32]. The rapid dispersal of the ARAF strains with the $\mathrm{TR}_{34} / \mathrm{L} 98 \mathrm{H}$ genotype among regions within Asia also supports the hypothesis that these strains have robust fitness in natural environments, with comparable or even higher fitness than that of wild-type strains $[21,23]$. However, we would like to note that the development of azole resistance in clinical ARAF isolates carrying mutations at other loci (i.e., the non-cyp51A gene) could result in a reduction of virulence [33].

\section{Perspectives}

The rapid spread of ARAF strains is jeopardizing the treatment of patients with Aspergillus diseases, ruling out the use of oral antifungals for these patients and leaving only the option of intravenous amphotericin B or echinocandins. Amphotericin B has significant detrimental side-effects and echinocandins are unable to completely kill or inhibit Aspergillus and, as such, they have only been licensed for salvage therapy of invasive aspergillosis. At present, antifungal susceptibility testing of $A$. fumigatus against azoles is not commonly performed and thus the overall threat of ARAF is not yet completely known. However, it would be beneficial to (i) have an active multi-azole susceptibility testing of A. fumigatus to monitor the extent of the problem, (ii) reduce agricultural use of triazole DMI fungicides, and (iii) use combination drug therapy when dealing with infections by $A$. fumigatus strains to limit the emergence of resistance. Indeed, the European Center for Disease Prevention and Control (http:// www.ecdc.europa.eu/en/publications/Publications/Forms/ECDC _DispForm.aspx?ID = 1064) recommends increased surveillance for clinical and environmental azole-resistant pathogens and to conduct field trials to study the impact of nonmedical azole use in the development of azole resistance in patients. A more judicious use of azoles in patients, in agriculture settings, and alternative strategies such as chemosensitization and/or a shift from the use of purely chemical methods to a more integrated crop management approach could help lower the dosage levels of fungicides in the environment and minimize the emergence and spread of ARAF.

Although there are substantial data suggesting that agricultural use of fungicides have driven the emergence and spread of multitriazole-resistant strains of $A$. fumigatus, conclusive evidence linking agricultural triazole fungicides to the emergence of $\mathrm{TR}_{34} / \mathrm{L} 98 \mathrm{H}$ or $\mathrm{TR}_{46} / \mathrm{Y} 121 \mathrm{~F} / \mathrm{T} 289 \mathrm{~A}$ genotypes in controlled field experiments is lacking. The definite evidence will help the regulatory authorities with formulation of policies to control the environmental-driven azole resistance. The two major types of azole-resistant mutations were first detected in 1998 and 2009 respectively and both are now 


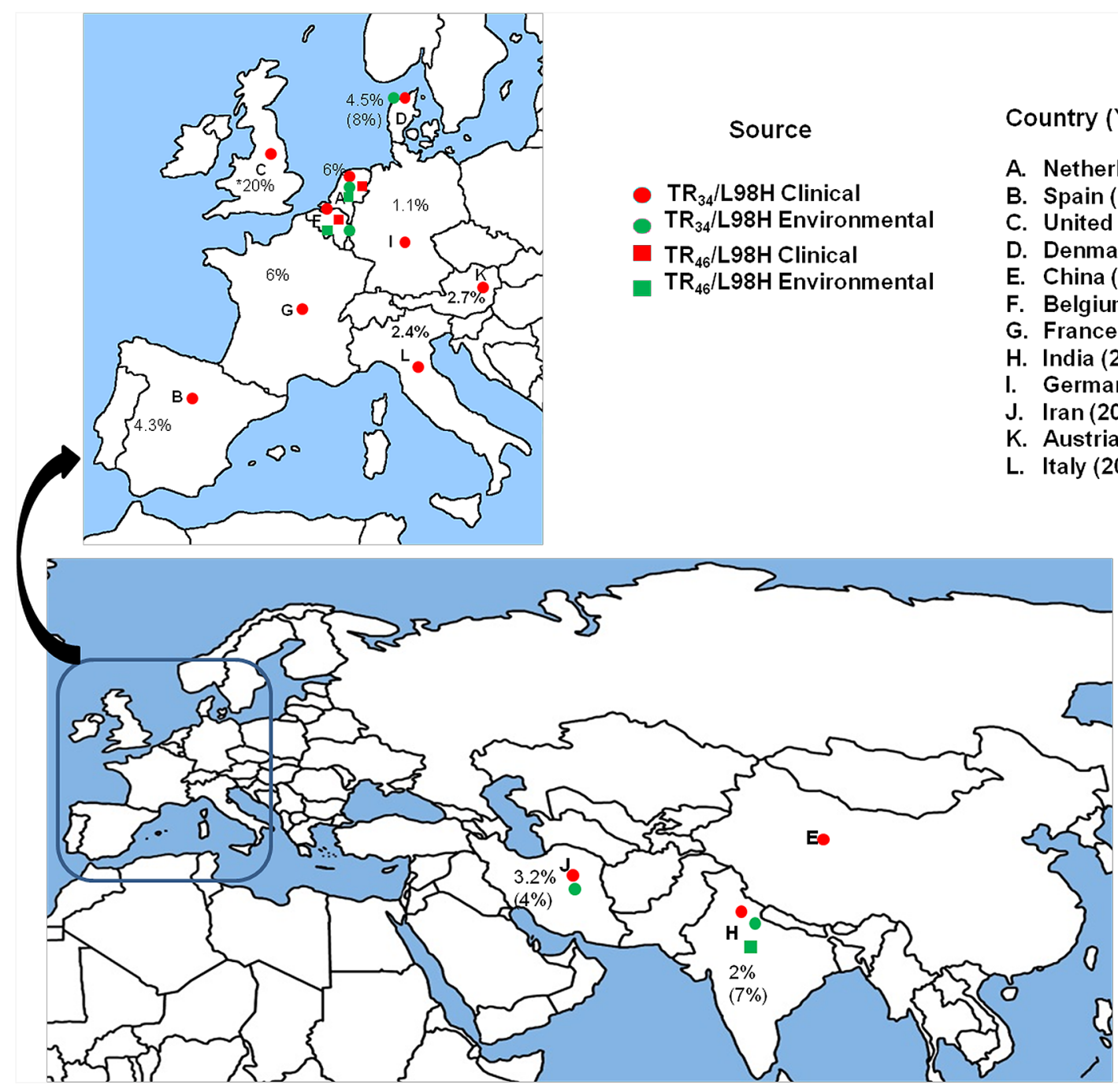

Figure 2. A global map depicting geographic distribution of multi-triazole-resistant clinical (red) and environmental (green) Aspergillus fumigatus strains carrying the $\mathrm{TR}_{\mathbf{3 4}} / \mathrm{L98H}$ (circle) and the $\mathrm{TR}_{\mathbf{4 6}} / \mathrm{Y} 121 \mathrm{~F} / \mathrm{T} 289 \mathrm{~A}$ mutations (square). Countrywide prevalence rates (\%) of $A$. fumigatus carrying $\mathrm{TR}_{34} / \mathrm{L} 98 \mathrm{H}$ are presented excepting the United Kingdom, where overall azole resistance is illustrated. The percent in parentheses denotes environmental prevalence rates.

doi:10.1371/journal.ppat.1003633.g002

spreading quickly (Figure 2). It is highly likely that other types of mutations conferring multiple azole resistance could emerge in the near future from environmental sources and spread among human populations.

\section{References}

1. Brown GD, Denning DW, Gow NA, Levitz SM, Netea MG, et al. (2012) Hidden killers: human fungal infections. Sci Transl Med 4:165rv13.

2. Denning D, Pleuvry A, Cole D (2013) Global burden of ABPA in adults with asthma and its complication chronic pulmonary aspergillosis in adults. Med Mycol 51: 361-370.

3. Lass-Flörl C (2011) Triazole antifungal agents in invasive fungal infections: a comparative review. Drugs 71: 2405-2419.

4. Van der Linden JWM, Arendrup MC, Verweij PE, SCARE network (2011) Prospective international surveillance of azole resistance in Aspergillus fumigatus: SCARE-Network. In: 51st Interscience Conference on Antimicrobial Agents and Chemotherapy (ICAAC); Sep 17-20; Chicago, IL. Abstract M-490. (SCARE-NETWORK)

5. Pfaller MA, Boyken L, Hollis R, Kroeger J, Messer S, et al. (2011) Use of epidemiological cutoff values to examine 9-year trends in susceptibility of Aspergillus species to the triazoles. J Clin Microbiol 49: 586-590.

\section{Acknowledgments}

Acknowledgement is due to Anupam Prakash and Cheshta Sharma, Research Scholars, Department of Medical Mycology, V. P. Chest Institute, for their assistance in figure preparation.

6. Krishnan-Natesan S, Swaminathan S, Cutright J, et al. (2010) Antifungal susceptibility pattern of Aspergillus fumigatus isolated from clinical specimens in Detroit Medical Center (DMC): rising frequency of high MIC of azoles (20032006). In: The 50th Interscience Conference on Antimicrobial Agents and Chemotherapy (ICAAC); Sep 12-15; Boston, MA, USA.

7. Chowdhary A, Kathuria S, Randhawa HS, Gaur SN, Klaassen CH, et al. (2012) Isolation of multiple-triazole-resistant Aspergillus fumigatus strains carrying the TR/L98H mutations in the $c y p 51 \mathrm{~A}$ gene in India. J Antimicrob Chemother 67: 362-366.

8. Seyedmousavi S, Hashemi SJ, Zibafar E, Zoll J, Hedayati MT, et al. (2013) Azole-resistant Aspergillus fumigatus, Iran. Emerg Infect Dis 19: 832-834.

9. Lockhart SR, Frade JP, Etienne KA, Pfaller MA, Diekema DJ, et al. (2011) Azole resistance in Aspergillus fumigatus isolates from the ARTEMIS global surveillance study is primarily due to the TR/L98H mutation in the cyp51A gene. Antimicrob Agents Chemother 55: 4465-4468. 
10. Bowyer P, Moore CB, Rautemaa R, Denning DW, Richardson MD (2011) Azole antifungal resistance today: focus on Aspergillus. Curr Infect Dis Rep 13: 485-491.

11. Snelders E, van der Lee HAL, Kuijpers J, Rijs AJMM, Varga J, et al. (2008) Emergence of azole resistance in Aspergillus fumigatus and spread of a single resistance mechanism. PLoS Med 5: e219. doi:10.1371/journal.pmed.0050219.

12. Van der Linden JWM, Snelders E, Kampinga GA, Rijnders BJA, Mattsson E, et al. (2011) Clinical implications of azole resistance in Aspergillus fumigatus, the Netherlands, 2007-2009. Emerg Infect Dis 17: 1846-1852.

13. Howard SJ, Cerar D, Anderson MJ, Albarrag A, Fisher MC, et al. (2009) Frequency and evolution of azole resistance in Aspergillus fumigatus associated with treatment failure. Emerg Infect Dis 15: 1068-1076.

14. Arendrup MC, Mavridou E, Mortensen KL, Snelders E, Frimodt-Møller N, et al. (2010) Development of azole resistance in Aspergillus fumigatus during azole therapy associated with change in virulence. PLoS ONE 5:e10080. doi:10.1371/ journal.pone.0010080.

15. Snelders E, Karawajczyk A, Verhoeven RJA, Venselaar H, Schaftenaar G, et al. (2011) The structure-function relationship of the Aspergillus fumigatus cyp51A $\mathrm{L} 98 \mathrm{H}$ conversion by site-directed mutagenesis: the mechanism of $\mathrm{L} 98 \mathrm{H}$ azole resistance. Fungal Genet Biol 48: 1062-1070.

16. Fraczek MG, Bromley M, Buied A, Moore CB, Rajendran R, et al. (2013) The cdr1B efflux transporter is associated with non-cyp51A mediated itraconazole resistance in Aspergillus fumigatus. J Antimicrob Chemother 68:1486-1496.

17. Mellado E, Garcia-Effron G, Alcazar-Fuoli L, Melchers WJ, Verweij PE, et al. (2007) A new Aspergillus fumigatus resistance mechanism conferring in vitro crossresistance to azole antifungals involves a combination of cyp51A alterations. Antimicrob Agents Chemother 51: 1897-904.

18. Snelders E, Karawajczyk, Schaftenaar G, Verweij PE, Melchers WJ (2010) Azole resistance profile of amino acid changes in Aspergillus fumigatus cyp51A based on protein homology modeling. Antimicrob Agents Chemother 54: 24252430 .

19. Snelders E, Huis In 't Veld RA, Rijs AJJM, Kema GHJ, Melchers WJ, et al. (2009) Possible environmental origin of resistance of Aspergillus fumigatus to medical triazoles. Appl Environ Microbiol 75: 4053-4057.

20. Denning DW, Park S, Lass-Flörl G, Fraczek MG, Kirwan M, et al. (2011) Highfrequency triazole resistance found in nonculturable Aspergillus fumigatus from lungs of patients with chronic fungal disease. Clin Infect Dis 52: 1123-1129.

21. Chowdhary A, Kathuria S, Xu J, Sharma C, Sundar G, et al. (2012) Clonal expansion and emergence of environmental multiple-triazole-resistant Aspergillus fumigatus strains carrying the $\mathrm{TR}_{34} / \mathrm{L} 98 \mathrm{H}$ mutations in the cyp51A gene in India. PLoS ONE 7: e52871. doi:10.1371/journal.pone.0052871.

22. Mortensen KL, Mellado E, Lass-Flörl C, Rodriguez-Tudela JL, Johansen HK, et al. (2010) Environmental study of azole-resistant Aspergillus fumigatus and other aspergilli in Austria, Denmark, and Spain. Antimicrob Agents Chemother 54: 4545-4549.

23. Badali H, Vaezi A, Haghani I, Yazdanparast SA, Hedayati MT, et al. (2013) Environmental study of azole-resistant Aspergillus fumigatus with $\mathrm{TR}_{34} / \mathrm{L} 98 \mathrm{H}$ mutations in the cyp51A gene in Iran. Mycoses. doi:10.1111/myc.12089. In press.

24. van der Linden JW, Camps SM, Kampinga GA, Arends JP, Debets-Ossenkopp YJ, et al. (2013) Aspergillosis due to voriconazole highly resistant Aspergillus fumigatus and recovery of genetically related resistant isolates from domestic homes. Clin Infect Dis 57: 513-520.

25. Vermeulen E, Maertens J, Schoemans H, Lagrou K (2012) Azole-resistant Aspergillus fumigatus due to $\mathrm{TR}_{46} / \mathrm{Y} 121 \mathrm{~F} / \mathrm{T} 289 \mathrm{~A}$ mutation emerging in Belgium, July 2012. Euro Surveill 17. pii:20326.

26. Chowdhary A, Sharma C, Kathuria S, Hagen F, Meis JF (2013) Azole-resistant Aspergillus fumigatus with the environmental TR46/Y121F/T289A mutation in India. J Antimicrob Chemother. doi:10.1093/jac/dkt397. In press.

27. Verweij PE, Kema GH, Zwaan B, Melchers WJ (2013) Triazole fungicides and the selection of resistance to medical triazoles in the opportunistic mould Aspergillus fumigatus. Pest Manag Sci 69: 165-170.

28. Serfling A, Wohlrab J, Deising HB (2007) Treatment of a clinically relevant plant-pathogenic fungus with an agricultural azole causes cross-resistance to medical azoles and potentiates caspofungin efficacy. Antimicrob Agents Chemother 51: 3672-3676.

29. Snelders E, Camps SMT, Karawajczyk A, Schaftenaar G, Kema GHJ, et al. (2012) Triazole fungicides can induce cross-resistance to medical triazoles in Aspergillus fumigatus. PLoS ONE 7: e31801. doi:10.1371/journal.pone.0031801.

30. Klaassen CH, Gibbons JG, Fedorova ND, Meis JF, Rokas A (2012) Evidence for genetic differentiation and variable recombination rates among Dutch populations of the opportunistic human pathogen Aspergillus fumigatus. Mol Ecol 21: $57-70$.

31. Mavridou E, Meletiadis J, Jancura P, Abbas S, Arendrup MC, et al. (2013) Composite survival index to compare virulence changes in azole-resistant Aspergillus fumigatus clinical isolates. PLoS ONE 8: e72280. doi:10.1371/ journal.pone.0072280.

32. Vale-Silva, Coste AT, Ischer F, Parker JE, Kelly SL, et al, (2012) Azole resistance by loss of function of the sterol $\Delta^{5},{ }^{6}$ - desaturase gene (ERG3) in Candida albicans does not necessarily decrease virulence. Antimicrob Agents Chemother 56: $1960-1968$.

33. Camps SMT, Dutilh BE, Arendrup MC, Rijs AJMM, Snelders E, et al. (2012) Discovery of a hapE mutation that causes azole resistance in Aspergillus fumigatus through whole genome sequencing and sexual crossing. PLoS ONE 7: e50034. doi:10.1371/journal.pone.0050034. 